\title{
IAMS Notice
}

Plans are still in process for the upcoming 15th IAMS Assembly, scheduled for 9th-15th July 2022 in Sydney, Australia, at Morling College, with the support of the Australian Association for Mission Studies. Because Australia's covid travel restrictions remain quite strict, with much uncertainty looking ahead, we urge you to refrain from making travel plans at this time unless full refunds can be secured.

We will communicate updates as soon as we have further news regarding dates, schedule, and any easing of travel restrictions.

Paul Kollman

IAMs President 